\title{
Current Approaches to the Treatment of Cerebral Vasospasm Following Subarachnoid Haemorrhage
}

\author{
a report by \\ R Webster Crowley, Aaron S Dumont and Neal F Kassell
}

Department of Neurological Surgery, School of Medicine, University of Virginia

DOI:10.17925/ENR.2008.03.01.40

Cerebral vasospasm following subarachnoid haemorrhage $(\mathrm{SAH})$ is the leading potentially treatable cause of morbidity and mortality in patients who experience the rupture of an intracranial aneurysm.1,2 A significant predictor of outcome in patients with aneurysmal SAH, cerebral vasospasm is radiographically present in up to $70 \%$ of patients and is clinically evident in 20-30\%.1,3 Annually, between seven and 20 people per 100,000 will experience a ruptured intracranial aneurysm. ${ }^{4-6}$ Fifty per cent of these patients will eventually die, while $15 \%$ will be rendered severely disabled. Only one-fifth to one-third of patients who experience a ruptured intracranial aneurysm will go on to have a moderate or good recovery. ${ }^{7,8}$ In large part, this poor prognosis is due to the significant death and disability associated with vasospasm. Approximately $50 \%$ of patients with symptomatic vasospasm will develop infarctions, and $15-20 \%$ will develop a disabling stroke or die of ischaemia. 9,10

Despite the clinical significance of cerebral vasospasm and the extensive research efforts dedicated towards elucidating its pathogenesis and therapy, vasospasm remains an incompletely understood clinical problem.

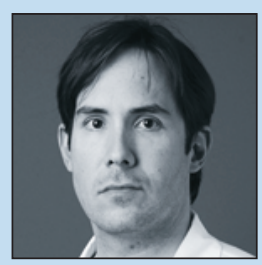

R Webster Crowley is a Neurosurgical Resident at the University of Virginia, and plans to pursue a career in cerebrovascular and endovascular surgery. Dr Crowley received his MD from The George Washington University. He enrolled in graduate school at the Medical College of Pennsylvania/Hahnemann University after completing his undergraduate training at the University of lowa, and completed his surgical internship at the University of Virginia.

E: rc9dd@virginia.edu

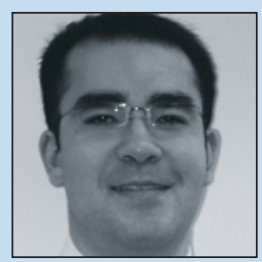

Aaron S Dumont is an Assistant Professor of Neurosurgery and Radiology, Co-Director of the Cerebrovascular and Skul Base Service and Neurosurgical Director of the Comprehensive Stroke Center at the University of Virginia. His clinical interests focus on cerebrovascular, endovascular and skull base surgery. His clinical and laboratory research concentrates on cerebrovascular disease and particularly on cerebral aneurysms, subarachnoid haemorrhage and cerebral vasospasm. Dr Dumont completed a fellowship in cerebrovascular and endovascular surgery at the University of Virginia.

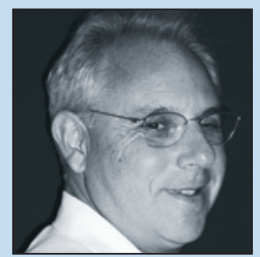

Neal F Kassell is a Professor of Neurosurgery at the University of Virginia, where he was Co-Chair until June 2006. He is a Director of the La Gesse Foundation, the Virginia National Bank and The Focused Ultrasound Surgery Foundation. He was a founder of Interax, Inc., the Virginia Neurological Institute and The Focused Ultrasound Surgery Foundation. Dr Kassell has been a recipient of the McKenzie Memorial Award of the Canadian Neurosurgical Society (CNS), the Grass Award of the Society of Neurological Surgeons (SNS) and the first Van Wagenen lectureship of the American Association of Neurological Surgeons (AANS).
Although progress has been made in the diagnosis and treatment of $\mathrm{SAH}$, the overall prognosis of patients with ruptured intracranial aneurysms remains poor. This article will review current strategies for the management of cerebral vasospasm.

\section{Treatment of Cerebral Vasospasm}

Several promising theories have developed from the intense research efforts aimed at cerebral vasospasm following SAH. However, as yet no individual theory has been able to completely explain its pathogenesis, which is complex and likely multifactorial; therefore, therapies directed at the treatment of cerebral vasospasm vary widely in both the intended target and the effect. These therapies can be broadly categorised into four groups according to the mechanism by which they are designed to reduce vasospasm, although no treatment has been found to be universally efficacious. These groups include therapies that prevent arterial narrowing, reverse arterial narrowing, enhance cerebral perfusion and protect against and rescue from cerebral ischaemia (see Table 1). While these groups are not mutually exclusive, and treatment options can potentially affect vasospasm through a variety of ways, they underscore the various ways in which the treatment of cerebral vasospasm can be approached. In order to treat vasospasm aggressively one must first treat the ruptured aneurysm itself, ideally within the first two days after aneurysmal rupture. ${ }^{4}$ Once the aneurysm is secured, the treating physician has the complete armamentarium available for the treatment of vasospasm.

\section{Removal of Blood Clot}

Cerebral vasospasm has been shown to arise from the presence of clot, specifically red blood cells (RBCs), ${ }^{11}$ within the subarachnoid space. A volume-dependent relationship exists between clot burden and subsequent risk of vasospasm. ${ }^{12-15}$ While conflicting opinions remain regarding the exact components within the clot that incite cerebral vasospasm, it is clear that the presence of clot is related to an increased risk of vasospasm.

Knowing that the presence of blood clot can incite cerebral vasospasm, it follows that reducing this clot burden may be an effective treatment for its prevention. Reducing the blood clot associated with $\mathrm{SAH}$, either with direct removal during surgery or with the delivery of catheter-infused tissue plasminogen activator (tPA) into subarachnoid cisterns, has been previously described in the literature. ${ }^{16-19}$ Indlay and colleagues ${ }^{19}$ conducted the only randomised trial looking at intra-operative recombinant ( $r$-tPA) therapy, with its injection into the basal subarachnoid cisterns following surgical management of ruptured intracranial aneurysms. They found that several groups tended towards lesser degrees of vasospasm when r-tPA was administered; however, the only statistically significant improvement was seen when it was administered in the setting of thick subarachnoid clots. A 
Table 1: Therapeutic Groups for the Prevention and Treatment of Cerebral Vasospasm

Prevention of arterial narrowing

- Clot removal

- Inhibition of the inflammatory response (anti-inflammatory agents, statins)

- Blockage of spasmogens (endothelin-1 antagonists, free radical scavengers)

- Disruption of contractile mechanism (prophylactic transluminal balloon angioplasty)

- Blockage of smooth-muscle-cell contraction (nimodipine, nicardipine, rho-kinase inhibitors, magnesium)

Reversal of arterial narrowing

- Balloon angioplasty

- Pharmacological angioplasty (verapamil, nicardipine, nimodipine, papaverine)

- Cerebral vasodilators (nitric oxide donors)

- Blockage of smooth-muscle-cell contraction (nimodipine, nicardipine, rho-kinase inhibitors, magnesium)

Enhancement of cerebral perfusion

- Triple-H therapy

Ischaemic protection and rescue

- Nitric oxide

later meta-analysis studied a total of 652 patients who were treated with intracisternal thrombolytics. ${ }^{16}$ This study concluded that, despite a lack of large, randomised, prospective trials, thrombolytic therapy provided a statistically significant beneficial effect. Their analysis found no difference between those who received intra-operative injections and those who received post-operative treatments. However, the dearth of well-designed prospective, randomised studies means that there are no consistent data supporting this routine practice.

\section{Blockage of Smooth-muscle-cell Contraction}

Therapies aimed at blocking the contraction of smooth muscle cells associated with cerebral vasospasm have shown promise. The calcium channel blocker nimodipine is now widely used for patients who experience the rupture of an intracranial aneurysm. This is largely due to clinical trials showing that the use of nimodipine for a period of 21 days after aneurysmal rupture improved outcome in patients with SAH. ${ }^{20,21}$ Although initially thought to prevent vasospasm, nimodipine does not appear to decrease the incidence of angiographic vasospasm; rather, its beneficial effects can be attributed to effects on the microcirculation or through neuroprotection.

On the other hand, another calcium channel blocker, nicardipine, has been shown to decrease the incidence of both symptomatic and angiographic cerebral vasospasm. ${ }^{22,23}$ However, unlike nimodipine it has not been shown to improve outcome, a finding that may be explained in part by the failure to account for use of rescue therapy. Both intra-arterial and intraventricular nicardipine have shown early therapeutic promise; however, further investigation is required. ${ }^{24,25}$

Magnesium has also been investigated in the setting of $\mathrm{SAH}$, albeit with mixed results. In 2005, van den Bergh and colleagues ${ }^{26}$ reported the results of their randomised, controlled trial examining intravenous magnesium sulphate in aneurysmal SAH. They observed a 34\% reduction in delayed cerebral ischaemia, and a reduction of poor outcome at three months by $23 \% .{ }^{26}$ Veyna et al. found that magnesium therapy did not reduce vasospasm rates; however they did see trends towards improved outcome. ${ }^{27}$ Primate studies have also found that magnesium did not prevent vasospasm. ${ }^{28}$ In summary, despite the lack of definitive evidence supporting its use, many centres advocate maintaining high levels of magnesium in patients with aneurysmal rupture due to the potential benefit with a low risk of adverse effects.

Other drugs that inhibit vascular smooth muscle contraction - such as rho-kinase inhibitors - have been studied in cerebral vasospasm. Fasudil is one such drug that may decrease angiographic vasospasm and computed tomography (CT) hypodensities, as well as decreasing symptomatic vasospasm and poor outcome. ${ }^{29,30}$ This drug has been approved for use in Japan, but has not been extensively investigated or implemented elsewhere.

\section{Statins}

Statins are another component of the medical therapy for cerebral vasospasm that have gained prevalence in the setting of SAH. Studies examining statins have shown varying effects in the setting of $\mathrm{SAH}$, including decreased incidence of vasospasm, reduced duration of severe vasospasm and a decreased mortality rate for patients with SAH. ${ }^{31-33}$ These beneficial effects are thought to be secondary to pleiotropic cholesterol-independent influences, including the downregulation of inflammatory processes and the upregulation of nitric oxide (NO) production via preserved endothelial nitric oxide synthase (eNOS) expression. ${ }^{31-33}$

In 2005, Lynch and colleagues ${ }^{32}$ studied the effects of simvastatin in patients with aneurysmal rupture and found that $80 \mathrm{mg}$ per day both attenuated radiographic vasospasm and delayed ischaemic neurological deficit. In a phase II randomised, placebo-controlled trial looking at the administration of pravastatin for patients with SAH, Tseng and colleagues ${ }^{31}$ observed a reduction of cerebral vasospasm and a decrease in delayed ischaemic neurological deficits. Their study showed the administration of statins was the only independent predictor of improved outcome at the time of hospital discharge. Recently, a retrospective cohort study performed at our institution looked at this practice, and found that the addition of statins to standard post-SAH care did not reduce the development of cerebral vasospasm or improve outcomes. ${ }^{34}$ However, further randomised controlled trials are needed to better elucidate its use.

\section{Endothelin-1 Inhibition}

Endothelin-1 (ET-1) is another potential contributor to SAH-induced vasospasm. ET-1 is a potent vasoconstrictor and is primarily produced by endothelial cells in response to ischaemia, although it can also be produced by neurons, astrocytes and activated leukocytes. ${ }^{35-37}$ The effects of ET-1 are mediated by two receptor subtypes, ETA and ETB, which activate a $G$ protein and a second messenger system. Located predominantly on smooth-muscle cells, ETA receptors mediate vasoconstriction and proliferation and appear to be critical in the development of cerebral vasospasm. ${ }^{35}$ Contributing to the implication of ET-1 in cerebral vasospasm is the finding that ET-1 levels are elevated in the plasma and cerebrospinal fluid of patients with $\mathrm{SAH}$, with elevated levels of ET-1 correlating with the persistence of cerebral vasospasm. ${ }^{38-40}$ Conversely, a decline in ET-1 levels has been observed in the absence of cerebral vasospasm. ${ }^{39}$

Efforts to discover a therapeutic solution to cerebral vasospasm have attempted to exploit this strong association between ET-1 and cerebral vasospasm following SAH. Studies have shown that the administration of ET-1 antagonists or inhibitors of the endothelin-converting enzyme 
(ECE) - which is responsible for activating endothelin - prevent vasospasm. ${ }^{35,41}$ One of these ETA receptor antagonists, clazosentan, is often considered the most promising drug that has been studied for the prevention or reversal of cerebral vasospasm. ${ }^{35}$ Clazosentan has been the subject of a phase Ilb clinical trial, the Clazosentan to Overcome Neurological iSChemia and Infarct OccUrring after Subarachnoid hemorrhage (CONSCIOUS-1) study. This was a double-blinded, randomised clinical trial that looked at the effects of clazosentan in cerebral vasospasm. The drug was found to decrease the incidence of severe vasospasm, delayed ischaemic neurological deficits and new infarcts seen on CT scans in a dose-dependent fashion. However, it did not show a reduction in patient mortality, although the study was underpowered for this end-point. ${ }^{42}$

Significant advances - with both open

surgical techniques and endovascular

techniques - have allowed the vast

majority of intracerebral aneurysms to

be treated effectively.

\section{Inhibition of Inflammatory Response}

The role of inflammation in the pathogenesis of cerebral vasospasm has also been closely investigated. ${ }^{36,43}$ Studies have shown the prominence of inflammation and leukocyte infiltration in the blood vessel walls following exposure to blood in the subarachnoid space. ${ }^{44,45}$ Additionally, leukocyte concentrations are elevated in the cerebrospinal fluid (CSF) of patients who develop SAH-related ischaemia, ${ }^{46}$ and adhesion molecules - such as ICAM-1, VCAM-1 and E-selectin - are similarly elevated in the CSF of patients with $\mathrm{SAH}$ and in blood vessel walls exposed to clot, in a time pattern that coincides with the natural course of cerebral vasospasm. ${ }^{36,47}$ Leukocytes can contribute to vasospasm in a variety of ways, including the promotion of free radical formation, ${ }^{48,49}$ the production of vasoactive substances such as leukotrienes and ET-137 or the consumption of NO.43 Cytokines may also be involved, with a profound alteration of their expression following SAH that correlates with the time course of vasospasm, ${ }^{50-52}$ and therapies targeting cytokines have demonstrated preliminary therapeutic efficacy. ${ }^{53,54}$

Despite the suspected role of inflammation in cerebral vasospasm, standard therapy for patients with aneurysmal SAH does not include antiinflammatory therapy. Several drugs directed at the inflammatory response have been used with varying levels of success, including nonsteroidal anti-inflammatory agents, FK-506, methylprednisolone, cyclosporine $\mathrm{A}$ and a host of other anti-inflammatory agents. ${ }^{36}$ The limited success of therapies directed at the inflammatory response is largely due to the fact that the critical constituents of the inflammatory response that contribute to vasospasm remain ill-defined. At this point there is no definitive role for anti-inflammatory therapy; however, it remains an area of great potential for future investigations.

\section{Bilirubin Oxidation Products}

One of the components of the subarachnoid clot that has been given particular attention in the potential pathogenesis of cerebral vasospasm has been bilirubin oxidation products (BOXes). BOXes cause smoothmuscle injury with subsequent vasoconstriction, and CSF levels have been noted to correlate with the time course of cerebral vasospasm.55,56 Increasing data have accrued supporting the role of BOXes in the pathogenesis of vasospasm in the setting of SAH and, as such, increasing attention has been paid to finding treatments that counteract their effects. ${ }^{55}$

As a component of the subarachnoid clot associated with cerebral vasospasm, perhaps the most effective method to counteract the potential effects of BOXes is the removal of clot, as discussed above. Other options include the prevention of bilirubin formation or the inactivation of bilirubin or BOXes. Although they have not been sufficiently studied, haeme oxygenase inhibitors have the potential to decrease the formation of bilirubin and the subsequent formation of BOXes. ${ }^{55}$ Free radical scavengers such as tirilazad have failed to produce benefit in the setting of $\mathrm{SAH},{ }^{57,58}$ although they remain an area of potential benefit.

\section{Prophylactic Angioplasty}

Prophylactic angioplasty is another therapeutic modality that has potential benefits for the prevention of cerebral vasospasm. There is little literature examining the use of angioplasty prophylactically for patients with aneurysmal rupture. Its use has been shown to prevent the development of vasospasm in patients who are considered at high risk of cerebral vasospasm. ${ }^{59}$ Currently, however, this is not a widely adopted practice due to the significant risks associated with prophylactic angioplasty, with as yet undetermined benefits. Recently, ZwienenbergLee and colleagues ${ }^{60}$ reported the results of their phase II multicentre randomised clinical trial looking at prophylactic transluminal balloon angioplasty (pTBA) in patients with Fisher Grade III SAH. They observed a statistically significant decrease in the need for therapeutic angioplasty in the patients who received PTBA; however, four patients (4.7\%) had their procedures complicated by vessel perforation, of whom three died. The study group concluded that PTBA did not have a significant impact on clinical course, and recommended that no further studies be performed at this point. ${ }^{60}$

\section{Triple-H Therapy}

Standard triple-H (hypervolaemia, hypertension and haemodilution) therapy remains a mainstay of the treatment of established cerebral vasospasm. This involves the induction of hypertension, hypervolaemia and mild haemodilution for patients demonstrated to be in cerebral vasospasm. ${ }^{61}$ Although opinions vary regarding the absolute necessity of haemodilution, the induction of hypertension and hypervolaemia with vasopressors in combination with volume expansion is considered the most effective way to quickly reverse the neurological deficits associated with cerebral vasospasm, with response rates approaching $70 \% .62,63$

\section{Nitric Oxide}

$\mathrm{NO}$ is a potent vasodilator that has been shown to play a significant role in the pathogenesis of cerebral vasospasm, largely because of the vasoconstriction - or rather the lack of vasodilation - seen with the depletion of NO.64 There are several mechanisms by which this depletion may occur in the setting of $\mathrm{SAH}$, including the downregulation or inhibition of NO synthases ${ }^{38,65-67}$ and the scavenging of NO by haemoglobin released during the breakdown of the subarachnoid clot. ${ }^{68,69}$ Further potentiating the effects of $\mathrm{NO}$ on cerebral vasospasm is 
the finding that NO may reverse the vasoconstriction seen with ET-1, with subsequent reduction of ET-1 levels. ${ }^{38,70}$

For these reasons, it follows that increasing the levels of NO in post-SAH patients would have potential in the prevention and treatment of cerebral vasospasm. Primate studies have shown that infusion of the NO donor sodium nitrite prevented cerebral vasospasm. ${ }^{71}$ However, further work is still required before an NO donor can be adopted for use in SAH patients. Currently, a phase I clinical trial examining sodium nitrite is under way, and subsequent clinical trials are being planned.

Another potential avenue to increase NO production in the setting of SAH is erythropoietin (EPO), which may aid in the prevention of cerebral vasospasm by increasing the phosphorylation of eNOS.72 EPO is also potentially neuroprotective, ${ }^{73,74}$ increasing its usefulness in patients with aneurysmal SAH.

\section{Endovascular Treatment for Established Vasospasm}

The endovascular treatment of established vasospasm can involve both medical and mechanical approaches. This includes transluminal balloon angioplasty of spastic segments of large intracranial arteries, 75,76 and the intra-arterial injection of vasoactive substances such as papaverine, verapamil, nicardipine and nimodipine. ${ }^{77-80}$ These interventions can be productive; however, in order to maximise their effectiveness they need to be administered in a timely fashion, and repeated treatments may be required.

\section{Conclusion}

Aneurysmal SAH is a potentially devastating condition with significant associated morbidity and mortality. Significant advances - with both open surgical techniques and endovascular techniques - have allowed the vast majority of intracerebral aneurysms to be treated effectively. Despite improved treatment for ruptured aneurysms, a significant proportion of patients who experience aneurysmal rupture will suffer additional injury secondary to cerebral vasospasm regardless of the effectiveness with which the aneurysm is treated. A variety of therapies have been investigated for the treatment of cerebral vasospasm pursuant to SAH, with varying degrees of success. While significant progress has been made and is continuing to be made for the treatment and prevention of vasospasm, the need for further investigation remains. Theories regarding the pathogenesis of cerebral vasospasm have continued to evolve, and therefore the treatment options have shown similar evolution. The role of ET-1, NO and BOXes has shown significant promise in elucidating the pathogenesis of cerebral vasospasm and, as such, therapies targeting these compounds have considerable promise for the future.
1. Kassell NF, Sasaki T, Colohan AR, Nazar G, Stroke, 1985; 16:562-72.

2. Kassell NF, Torner JC, Haley ECJ, et al., J Neurosurg, 1990;73: 18-36.

3. Weir BK, Macdonald RL, Stoodley M, Acta Neurochir Suppl (Wien), 1999;72:27-46.

4. Kassell NF, Torner JC, Stroke, 1984;15: 566-70.

5. Schievink W, N Engl J Med, 1997;336: 28-40.

6. Ronkainen A, Miettinen H, Karkola K, et al., Stroke, 1998;29: 359-62.

7. Rosenorn J, Eskesen V, Schmidt K, et al., Br J Neurosurg, 1987;1: 33-46.

8. Saveland $H$, Sonesson $B$, Ljundggren $B$, et al., J Neurosurg, 1986;64:191-6.

9. Mayberg MR, Batjer HH, Dacey RGJ, et al., Stroke, 1994;25: 2315-28.

10. Haley EC, Kassell NF, Torner JC, Stroke, 1992;23:205-14.

11. Okada T, Harada T, Bark DH, Mayberg MR, Neurosurgery, 1990;27:349-56.

12. Fisher CM, Kistler JP, Davis JM, Neurosurgery, 1980;6:1-6.

13. Macdonald RL, Weir B, Stroke, 1991;22:971-82.

14. Pluta RM, Afshar JK, Boock RJ, Oldfield EH, J Neurosurg, 1998;88:557-61.

15. Reilly C, Amidei C, Tolentino J, et al., J Neurosurg, 2004;101: 255-61.

16. Amin-Hanjani S, Ogilvy CS, Barker FG II, Neurosurgery, 2004;54: 326-34, discussion 334-5.

17. Handa Y, Weir BK, Nosko M, et al., J Neurosurg, 1987;67:558-64.

18. Kawamoto S, Tsutsumi K, Yoshikawa G, et al., J Neurosurg, 2004; 100:236-43.

19. Findlay JM, Kassell NF, Weir BK, et al., Neurosurgery, 1995;37: 168-76, discussion 177-68

20. Allen GS, Ahn HS, Preziosi TJ, et al., N Engl J Med, 1983;308: 619-24

21. Barker FG, Ogilvy CS, J Neurosurg, 1996;84:405-14.

22. Haley EC Jr, Kassell NF, Torner JC, J Neurosurg, 1993;78:537-47.

23. Haley EC Jr, Kassell NF, Torner JC, J Neurosurg, 1993;78:548-53.

24. Tejada JG, Taylor RA, Ugurel MS, et al., ANNR Am J Neuroradiol, 2007;28: 844-8.

25. Goodson K, Lapointe M, Monroe T, Chalela JA, Neurocrit Care, 2008:8(2):247-52.

26. van den Bergh WM, Algra A, van Kooten F, et al., Stroke, 2005;36:1011-15

27. Veyna RS, Seyfried D, Burke DG, et al., J Neurosurg, 2002;96: 510-14.

28. Macdonald RL, Curry DJ, Aihara Y, et al., J Neurosurg, 2004;100:
$106-10$.

29. Shibuya M, Suzuki Y, Sugita K, et al., J Neurosurg, 1992;76:571-7.

30. Zhao J, Zhou D, Guo J, et al., Neurol Med Chir (Tokyo), 2006;46: 421-8.

31. Tseng M-Y, Czosnyka M, Richards H, et al., Stroke, 2005;36: 1627-32.

32. Lynch JR, Wang H, McGirt MJ, et al., Stroke, 2005;36:2024-6.

33. Tseng MY, Hutchinson PJ, Czosnyka M, et al., Stroke, 2007;38: 1545-50.

34. Kramer AH, Gurka MJ, Nathan B, et al., Neurosurgery, 2008;62: 422-7, discussion 427-30.

35. Chow M, Dumont AS, Kassell NF, Neurosurgery, 2002;51: 1333-41, discussion 1342.

36. Dumont AS, Dumont RJ, Chow MM, et al., Neurosurgery, 2003;53: 123-35.

37. Fassbender K, Hodapp B, Rossol S, et al., Stroke, 2000;31: 2971-5.

38. Pluta RM, Pharmacol Ther, 2005;105:23-56.

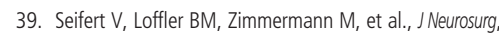
1995:82:55-62.

40. Juvela S, J Neurosurg, 2000:92:390-400

41. Sasaki T, Kassell NF, Neurosurg Clin N Am, 1990;1:451-63.

42. Macdonald RL, Stroke, 2007;38:a453-a607.

43. Provencio JJ, Vora N, Semin Neurol, 2005;25:435-44.

44. Handa Y, Kabuto M, Kobayashi H, et al., Neurosurgery, 1991;28: 542-9.

45. Mayberg MR, Okada T, Bark DH, Neurosurg Clin N Am, 1990;1: 417-32.

46. Spallone A, Acqui M, Pastore FS, Guidetti B, Surg Neurol, 1987;27: 253-8.

47. Polin RS, Bavbek M, Shaffrey ME, et al., J Neurosurg, 1998;89: 559-67.

48. Sullivan GW, Sarembock JJ, Linden J, J Leukoc Biol, 2000;67 591-602.

49. Grisham MB, Granger DN, Lefer DJ, Free Rad Biol Med, 1998;25: 404-33.

50. Fassbender K, Hodapp B, Rossol S, et al., I Neurol Neurosurg Psychiatry, 2001;70:534-7.

51. Hirashima Y, Nakamura S, Endo $S$, et al., Neurochem Res, 1997;22:1249-55.

52. Takizawa T, Tada T, Kitazawa K, et al., Neurol Res, 2001;23: 724-30.

53. Bowman G, Bonneau RH, Chinchilli VM, et al., Neurocrit Care, 2006;5:222-9.

54. Bowman G, Dixit S, Bonneau RH, et al., Neurosurgery, 2004:54: 719-25, discussion 725-6.
55. Clark J, Sharp F, J Cereb Blood Flow Metab, 2006;26:1223-33.

56. Pyne-Geithman GJ, Morgan CJ, Wagner K, et al., J Cereb Blood Flow Metab, 2005;23:23

57. Haley EC Jr, Kassell NF, Apperson-Hansen C, et al., J Neurosurg, 1997:86:467-74.

58. Weyer GW, Nolan CP, Macdonald RL, Neurosurg Focus, 2006:21:E8.

59. Muizelaar JP, Zwienenberg M, Rudisill NA, Hecht ST, J Neurosurg, 1999;91:51-8.

60. Zwienenberg-Lee M, Hartman J, Rudisill N, et al., Stroke, 2008; epub ahead of print.

61. Sen J, Belli A, Albon H, et al., Neurol, 2003;2:614-21.

62. Finn SS, Stephensen SA, Miller CA, et al., J Neurosurg, 1986;65: 48-62.

63. Kassell NF, Peerless SJ, Durward QJ, et al., Neurosurgery, 1982;11:337-43

64. Sehba FA, Schwartz AY, Chereshnev I, Bederson JB, I Cereb Blood Flow Metab, 2000;20:604-11.

65. Hino A, Tokuyama Y, Weir B, et al., Neurosurgery, 1996;39:562-7, discussion 567-8.

66. Pluta RM, Thompson BG, Dawson TM, et al., J Neurosurg, 1996;84:648-54.

67. Jung CS, Iuliano BA, Harvey-White J, et al., J Neurosurg, 2004;101: 836-42.

68. Goretski J, Hollochert T, J Biol Chem, 1988;263:2316-23.

69. Ignarro L, Annu Rev Pharmacol Toxicol, 1990;30:535-60.

70. Thomas JE, Nemirovsky A, Zelman V, Giannotta SL, Neurosurgery, 1997:40:1245-9.

71. Pluta RM, Dejam A, Grimes G, et al., JAMA, 2005;293: 1477-84.

72. Santhanam AVR, Smith LA, Akiyama M, et al., Stroke, 2005;36: 2731-7.

73. Alafaci C, Salpietro F, Grasso G, et al., Eur J Pharmacol, 2000; 406:219-25.

74. Buemi M, Grasso G, Corica F, et al., Eur J Pharmacol, 2000;392: $31-4$.

75. Higashida RT, Hieshima GB, Tsai FY, et al., ANNR, 1987:8:745-9.

76. Bejjani GK, Bank WO, Olan WJ, Sekhar LN, Neurosurgery, 1998:42:979-87.

77. Polin RS, Hansen CA, German P, et al., Neurosurgery, 1998;42:1256-64, discussion 1264-57.

78. Hanggi D, Turowski B, Beseoglu K, et al., AJNR Am J Neuroradiol, 2008; epub ahead of print.

79. Keuskamp J, Murali R, Chao KH, J Neurosurg, 2008;108:458-63.

80. Feng L, Fitzsimmons BF, Young WL, et al., AINR Am J Neuroradiol, 2002;23:1284-90. 